\title{
Calculation of thermodynamic properties of liquid Fe-Ln alloys
}

\author{
Natalia V. Golovata*, Natalia V. Kotova, Natalia I. Usenko \\ Department of Chemistry, Taras Shevchenko National University of Kyiv, Volodymyrska Street, \\ 64/13, Kyiv 01601, Ukraine \\ contacting e-mail nata.hoff@mail.ru
}

Keywords: liquid alloys; iron; lanthanides; thermodynamic properties calculation; "surrounded atom" model.

This paper presents the results of estimation of thermodynamic properties of binary Fe-Ln systems which characterized by small or moderate energy of component interaction in liquid state. The good agreement between enthalpies of mixing calculated in "surrounded atom" approximation and experimentally obtained enthalpies of mixing is observed for all investigated systems including $\mathrm{Fe}-\mathrm{Pr}$ one, in which sign variable values of heat effects were determined. The method of estimation of excess Gibbs energies of liquid Fe-Ln alloys is proposed and the results of such estimation are given for a number of systems including those experimentally unexplored yet.

\section{Introduction}

Binary and ternary alloys containing iron and lanthanides (Ln) are promising materials because of its possible technical application as amorphous, magnetic materials and materials for hydrogen batteries. Reliable thermodynamic information for liquid Fe-Ln alloys is necessary to calculate phase equilibria in multicomponent systems, many of which are practically important because of unique technological properties.

\section{Results and discussion}

Recently, the enthalpies of mixing of liquid alloys of iron with some lanthanides $(\mathrm{Pr}$, $\mathrm{Nd}, \mathrm{Gd}, \mathrm{Tb}$, Dy and Lu) have been obtained (Ref. [1]) by high-temperature isoperibolic calorimetry at $1550-1950 \mathrm{~K}$ through the entire composition range.

It was shown that the heat effects of alloy formation in the $\mathrm{Fe}-\mathrm{Pr}$ system were sign variable values with small extremes: negative values with minimum enthalpy of mixing at $\mathrm{x}_{\mathrm{Pr}}=0.22\left(\Delta_{\mathrm{m}} H_{\text {min }}=-0.35 \pm 0.10 \mathrm{~kJ} \cdot \mathrm{mol}^{-1}\right)$ and positive one with maximum at $\mathrm{x}_{\mathrm{Pr}}=0.76$ $\left(\Delta_{\mathrm{m}} H_{\max }=0.75 \pm 0.24 \mathrm{~kJ} \cdot \mathrm{mol}^{-1}\right)$.

The integral enthalpy of mixing of liquid $\mathrm{Fe}-\mathrm{Nd}$ alloys is characterized by small negative values through the whole composition range with minimum value $\left(\Delta_{\mathrm{m}} H_{\min }=-1.08 \pm 0.35\right.$ $\left.\mathrm{kJ} \cdot \mathrm{mol}^{-1}\right)$ at $\mathrm{x}_{\mathrm{Nd}}=0.40$.

The process of alloy formation of iron with heavy lanthanides is accompanied by more significant exothermic effects, increasing 
through the lanthanide series from the $\mathrm{Fe}-\mathrm{Gd}$ system $\left(\Delta_{\mathrm{m}} H_{\text {min }}=-4.74 \pm 0.40 \mathrm{~kJ} \cdot \mathrm{mol}^{-1}\right.$ at $\mathrm{x}_{\mathrm{Gd}}=0.45 ; \mathrm{T}=1833 \mathrm{~K}$ ) to the $\mathrm{Fe}-\mathrm{Lu}$ system $\left(\Delta_{\mathrm{m}} H_{\min }=-11.58 \pm 0.50 \mathrm{~kJ} \cdot \mathrm{mol}^{-1}\right.$ at $\mathrm{x}_{\mathrm{Lu}}=0.35$; $\mathrm{T}=1950 \mathrm{~K})$.

An increase in the exothermic heat effects is associated mainly with a decrease in size mismatch of the components due to the effect of the lanthanide contraction.

The direct high temperature experimental determination of the thermodynamic properties of such rather refractory alloys in liquid state is difficult, labour- and energy-consuming process. Therefore, the development of model calculation methods for prediction of thermodynamic properties of metal systems at high temperatures is important current task. Such methods allow us to obtain rather reliable thermodynamic information for binary systems basing on few experimental points without carrying out the experiments through the whole composition interval.

Thus, a method of calculating of the enthalpies, excess entropies and excess Gibbs energies of mixing of binary liquid alloys has been developed at the Department of Physical Chemistry of Taras Shevchenko National University of Kyiv [2]. This method is based on the theory of "surrounded atom" [3] and allows to simulate above-mentioned properties through the entire concentration range in the course of iterative procedure using either two experimental values of the integral enthalpy
$\Delta_{\mathrm{m}} H$ or the partial enthalpies of mixing of both components at infinite dilution $\Delta \bar{H}_{\mathrm{i}}^{\infty}$. Using the "surrounded atom" method we have simulated the integral enthalpies of mixing $\left(\Delta_{\mathrm{m}} H_{\text {calc }}\right)$ for investigated Fe-Ln melts for entire range of concentrations and compared these values with experimentally obtained results $\left(\Delta_{\mathrm{m}} H\right)$. Comparison of integral enthalpy values obtained experimentally and simulated through the whole concentration range is given in Figure 1.

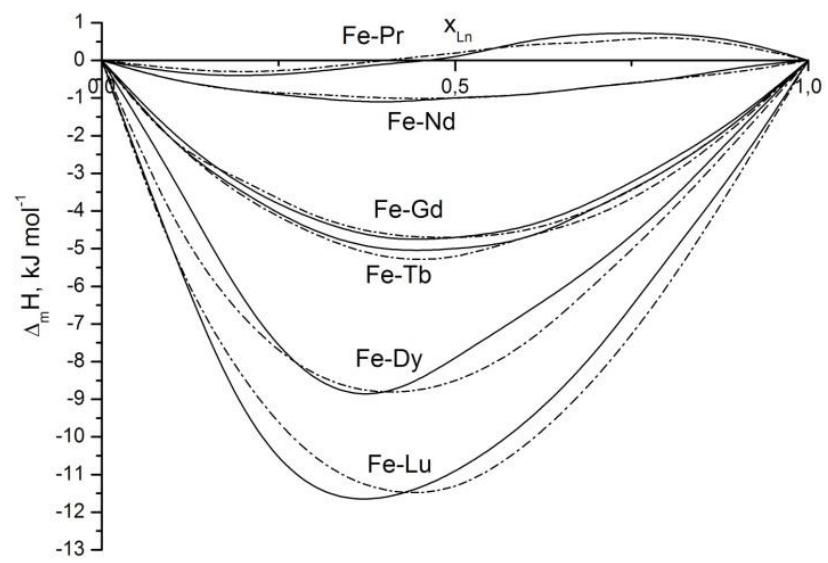

Figure 1. Integral enthalpies of mixing of liquid Fe-Ln alloys: values obtained experimentally (solid lines) and simulated through the whole concentration range (dashed lines)

The data on the figure indicate quite reasonable agreement between experimentally obtained values and simulated ones. Thus, the relative error $\left(\sigma=\left(\frac{\left|\Delta_{\mathrm{m}} H_{\text {calc }}-\Delta_{\mathrm{m}} H\right|}{\Delta_{\mathrm{m}} H}\right) \cdot 100 \%\right)$ averages 7.0, 2.4, 3.4, 7.6 and $6.3 \%$ for the Fe$\mathrm{Nd}, \mathrm{Fe}-\mathrm{Gd}, \mathrm{Fe}-\mathrm{Tb}, \mathrm{Fe}-\mathrm{Dy}$ and Fe-Lu systems, respectively. Such values do not exceed an ordinary error of calorimetric experiment, which is generally considered to be $10 \%$. The Fe-Pr 
system to a certain extent stands out from this row: the relative difference between the calculated and experimental results is about 40 $\%$. However, this is a good result for the description of a rather complex form of the concentration dependence with small sign variable values. Then for the investigated systems the partial enthalpies of mixing of both components at infinite dilution were calculated in "surrounded atom" approximation and were compared with the experimentally obtained in Ref. [1] values (see Table 1).

Table 1. The partial enthalpies of mixing at infinite dilution of iron and lanthanides, determined from experiment and calculated by «surrounded atom» model, $\mathrm{kJ} \cdot \mathrm{mol}^{-1}$

\begin{tabular}{|c|c|c|c|c|}
\hline \multirow{2}{*}{ System } & \multicolumn{2}{|c|}{$\Delta \bar{H}_{\mathrm{Ln}}^{\infty}$} & \multicolumn{2}{c|}{$\Delta \overline{\boldsymbol{H}}_{\mathrm{Fe}}^{\infty}$} \\
\cline { 2 - 5 } & Experiment & Calc. & Experiment & Calc. \\
\hline $\mathrm{Fe}-\mathrm{Pr}$ & $-3.6 \pm 0.8$ & -3.4 & $5.4 \pm 1.8$ & 5.6 \\
\hline $\mathrm{Fe}-\mathrm{Nd}$ & $-5.8 \pm 1.6$ & -5.7 & $-2.0 \pm 1.4$ & -2.6 \\
\hline $\mathrm{Fe}-\mathrm{Gd}$ & $-22.0 \pm 3.6$ & -21.6 & $-15.6 \pm 2.8$ & -16.8 \\
\hline $\mathrm{Fe}-\mathrm{Tb}$ & $-23.5 \pm 2.5$ & -23.9 & $-16.8 \pm 3.0$ & -17.8 \\
\hline $\mathrm{Fe}-\mathrm{Dy}$ & $-30.1 \pm 3.0$ & -37.8 & $-20.8 \pm 2.7$ & -20.1 \\
\hline $\mathrm{Fe}-\mathrm{Lu}$ & $-54.4 \pm 3.6$ & -57.9 & $-28.0 \pm 3.8$ & -32.1 \\
\hline
\end{tabular}

It is evident that in many cases the calculated values are within the confidence intervals of the experimentaly determined values. The exception is the Fe-Dy system, which has a special domed form of the concentration dependence of the partial enthalpies of mixing of lanthanide [1]. Such form may be associated with the influence of congruently melting $\mathrm{Fe}_{17} \mathrm{Dy}_{2}$ compound. Compounds of the same stoichiometry $\mathrm{Fe}_{17} \mathrm{Ln}_{2}$ in other $\mathrm{Fe}-\mathrm{Ln}$ systems melt by peritectic reactions [4].

Thus, the application of the "surrounded atom" model to simulate the enthalpies of mixing of the liquid metal systems of the same type is rather successful.

Moreover, the surrounded atom model allows us to calculate not only enthalpies of mixing, but also excess entropies and excess Gibbs energies of mixing for the investigated systems. Indeed, as we have the experimental values of partial enthalpies of mixing at infinite dilution we can estimate partial excess entropies of mixing according to Kubaschewski regularity [5]. "Surrounded atom" approach makes possible to calculate concentration dependences of excess Gibbs energies of mixing through the whole composition range using the data on the enthalpies and entropies of mixing at infinite dilution. The calculated values can be considered as rather appropriate estimation of $\Delta_{\mathrm{m}} G^{\mathrm{ex}}$ in the case of binary systems with small or moderate component interaction. For example, the results of the calculation of $\Delta_{\mathrm{m}} G^{\mathrm{ex}}$ and $\Delta_{\mathrm{m}} S^{\mathrm{ex}}$ values for the Fe-Dy system are given in Figure 2.

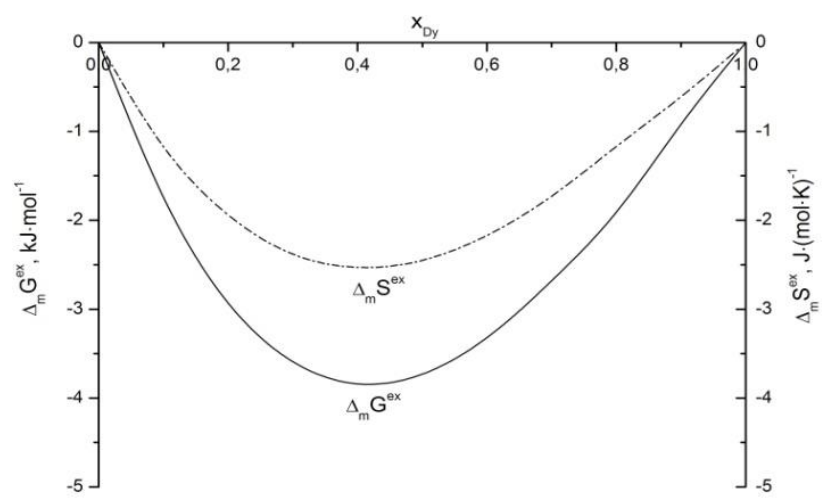

Figure 2. The concentration dependences of excess Gibbs energy of mixing and excess entropy of mixing of liquid Fe-Dy alloys 
Furthermore, we can estimate $\Delta_{\mathrm{m}} G^{\mathrm{ex}}$ values not only for investigated systems but also for those of the same Fe-Ln series that are unexplored experimentally yet. Such estimation is based on the regular increase of exothermic values of integral and partial enthalpies of mixing through the lanthanide row. Table 2 presents the data on concentration dependencies of $\Delta_{\mathrm{m}} G^{\mathrm{ex}}$ in polynomial series form for a number of $\mathrm{Fe}-\mathrm{Ln}$ systems (where $\mathrm{Ln}$ is the metal of Gd subgroup), both for investigated and unexplored ones.

In order to simulate $\Delta_{\mathrm{m}} G^{\mathrm{ex}}$ values for unexplored systems we need the initial data on partial mixing enthalpies of the components at infinite dilution. These values were estimated using linear fit of experimentally obtained $\Delta \bar{H}_{\mathrm{i}}^{\infty}$ values of the components through the lanthanide series.

Table 2. The concentration dependences of excess Gibbs energies of mixing calculated by «surrounded atom» model, $\mathrm{kJ} \cdot \mathrm{mol}^{-1}$

\begin{tabular}{|c|c|}
\hline System & \multicolumn{1}{|c|}{ Polynomial concentration dependences of $\Delta_{\mathrm{m}} G^{\mathrm{ex}}\left(x=x_{\mathrm{Ln}}\right)$} \\
\hline $\mathrm{Fe}-\mathrm{Gd}$ & $x(1-x)\left(-20.07+38.61 x-14.94 x^{2}+370.79 x^{3}-434.77 x^{4}+190.11 x^{5}\right)$ \\
\hline $\mathrm{Fe}-\mathrm{Tb}$ & $x(1-x)\left(-19.97+14.53 x-1.48 x^{2}-10.15 x^{3}+10.39 x^{4}\right)$ \\
\hline $\mathrm{Fe}-\mathrm{Dy}$ & $x(1-x)\left(-20.81+18.53 x-53.59 x^{2}+163.00 x^{3}-215.21 x^{4}+101.05 x^{5}\right)$ \\
\hline $\mathrm{Fe}-\mathrm{Ho}$ & $x(1-x)\left(-22.13-1.41 x+140.67 x^{2}-581.47 x^{3}+1150.83 x^{4}-1110.56 x^{5}+418.69 x^{6}\right)$ \\
\hline $\mathrm{Fe}-\mathrm{Er}$ & $x(1-x)\left(-23.30-15.57 x+283.86 x^{2}-1191.67 x^{3}+2389.63 x^{4}-2297.08 x^{5}+849.93 x^{6}\right)$ \\
\hline $\mathrm{Fe}-\mathrm{Tm}$ & $x(1-x)\left(-23.56+14.87 x-3.16 x^{2}-6.40 x^{3}+5.98 x^{4}\right)$ \\
\hline $\mathrm{Fe}-\mathrm{Lu}$ & $x(1-x)\left(-30.91+103.69 x-454.16 x^{2}+1109.62 x^{3}-1457.13 x^{4}+979.08 x^{5}-265.54 x^{6}\right)$ \\
\hline
\end{tabular}

